\title{
DINAMISMO CONSTRUCCIONAL EN EL LENGUAJE INFANTIL Y TEORÍA LINGÜÍSTICA*
}

\author{
Milagros Fernández Pérez \\ Universidad de Santiago de Compostela \\ Grupo de investigación koiné <www.usc.es/koine/> \\ femagos@usc.es
}

\begin{abstract}
Resumen
The development of child language shows particular features which do not fit in adult language patterns. The idiosyncrasy of child language in its different stages has not always been paid attention to. Most of the theories approach child language as though it was a fully conformed system. There are, however, several approaches which study child language as a particular code, focusing its particular emergency.
\end{abstract}

\section{Introducción}

Cuando se trata de lenguaje infantil, el enfoque habitual ha sido o bien aplicar teorías lingüísticas o psicolingüísticas generales con objeto de corroborarlas en su adecuación empírica. O bien, y muchas veces de manera simultánea con tal enfoque, abordar de forma atomista la adquisición del lenguaje analizándolo en unidades y procedimientos del lenguaje adulto. En este modo de proceder, además de problemas conceptuales ligados a lo que es en realidad un proceso dinámico y evolutivo, hay, sobre todo, un grave inconveniente metodológico. De entrada, aproximarse al lenguaje infantil desde el prisma de los productos característicos del lenguaje adulto no se compadece con las peculiaridades propias de las distintas etapas de desarrollo de la lengua. Pocas garantías tendrán las contrastaciones de las teorías si se fuerzan los datos del lenguaje infantil o si su descripción está tamizada a través del código definitivamente conformado.

Para recomponer de modo aceptable la perspectiva sobre la adquisición de la lengua se hace imprescindible reflexionar acerca del papel de las teorías lingüísticas al uso. Y desde esas consideraciones meditadas se valorarán los procedimientos analíticos por su idoneidad para ajustarse a la descripción y al pormenor de las propiedades del lenguaje infantil. La relevancia de las teorías lingüísticas en el tema del desarrollo del lenguaje conviene ponderarla tanto por su capacidad para integrar la diversidad y el dinamismo, como por su poten-

\footnotetext{
* Este trabajo se inscribe en el marco del proyecto Evaluación lingüistica de déficit que inciden en gramática, que ha recibido soporte económico de la Xunta de Galicia (Dirección Xeral de Investigación e Desenvolvemento), PGIDIT02PXIC20403PN; y del Ministerio de Ciencia y Tecnología, BFF2001-3234-C02-01.
} 
cia para sobre los datos heterogéneos de adquisición sistematizar principios y características que definan los códigos en cada una de las fases evolutivas.

Desde hace ya algunos años la denominación de adquisición del lenguaje se ha visto sustituida por la de adquisición de la lengua, o, de modo más detallado y explícito, por la de proceso de desarrollo de la lengua en el niño. Síntoma de la relevancia de las características idiomáticas y tipológicas en la dinámica de la adquisición. E indicador claro de la importancia relativa de teorías representacionales cuyo cometido es simular en un esquema o modelo EL PROCESO, como si fuese único y constante en sus derroteros. La importancia de los factores del entorno lingüístico y social y el peso de características individuales no sólo han sido revulsivos en el tratamiento del desarrollo ontogenético en su dimensión lingüística, sino que han incidido también de manera notable en cómo abordar la evolución cognitiva y el desarrollo psicológico en el niño. En cualquiera de las vertientes lo prioritario son los DATOS DE ADQUISICIÓN. Son necesarias pues teorías instrumentales que faciliten el acceso a los materiales que se observan o con los que se experimenta, y que canalicen las descripciones necesarias en cada momento. Teorías analíticas suficientemente flexibles y versátiles que puedan dar cabida a materiales lingüísticos infantiles sin acomodarlos o someterlos al modelo adulto.

Este artículo quiere ser una contribución al trazado de un camino en el estudio de la lengua infantil respetando sus peculiaridades. Para lo que hay que empezar a desterrar algunos tópicos y a valorar los planteamientos metodológicos y teóricos en su función clarificadora y descriptiva en este ámbito. Comenzaré borrando algunos lugares comunes para después contrastar las aproximaciones teóricas a cuestiones de desarrollo gramatical como las "clases" de verbos manejados, el número de participantes en las construcciones utilizadas, o las pautas que miden la habilidad comunicativa.

\section{Desterrando algunos tópicos}

Las tentativas de comprender la emergencia de la habilidad comunicativa en un período de tiempo relativamente corto y aún cuando el estímulo sea pobre han dado lugar a interpretaciones teóricas excesivamente abarcadoras. Los modelos han incluido el proceso de adquisición lingüística y el desarrollo cognitivo en muchas ocasiones sin diferenciarlos. Han obviado los requisitos del progreso en la comprensión y las exigencias del avance en la faceta expresiva, como si las fases fuesen parejas o idénticas. Y han identificado la adquisición con la aparición de la sintaxis, como si el desarrollo del componente fónico no fuese condición para avanzar en el manejo de palabras y, con éstas, construir estructuras. Como telón de fondo, el código adulto conformado es el que sirve de referencia para aproximarse y valorar el lenguaje infantil ${ }^{\prime}$. Y como principio rector, la idea de que saber hablar es saber utilizar LA GRAMÁTICA, como si la habilidad lingüística no fuese graduable y distinta entre los

1 Autores como D. Slobin muestran su perplejidad cuando se pretende hallar en la lengua los procedimientos que metodológicamente se han diseñado para analizarla:

The modules that are postulated often have names that evoke suspicion: they are the names of our own academic fields (linguistics, mathematics, physics, biology) or subfields (closed-class morphemes, grammaticizable notions). Could God or evolution have anticipated the academic and intellectual organization of late twentieth-century America? (Slobin, 2001: 442-443) 
individuos y como si la comunicación no fuese el elemento clave para ponderar el desarrollo lingüístico y sus efectos.

Los planteamientos universalistas sobre la adquisición de la lengua han de ser abandonados si se quieren diseñar modelos realistas que reflejen la dinámica del complejo proceso de emergencia de la habilidad. En primer lugar, hay que distinguir teorías del desarrollo cognitivo y teorías de la adquisición lingüística. Si bien investigadores de prestigio en el campo de la Psicolingüística -como J. Piaget, L. Vygotsky, R. Brown o, más recientemente, D. Slobin y M. Bowerman- han insistido en las peculiaridades del proceso cognitivo, no obstante ha habido concepciones sobre la adquisición de la lengua arraigadas en bases psicológicas y mentales siendo el lenguaje un módulo cognitivo y tomando la Lingüística como una rama de la Psicolingüística -visión ésta propia de la línea chomskiana y reflejada en el propio N. Chomsky, J. Fodor o S. Pinker-. En líneas generales, la identificación o el paralelismo entre los procesos de maduración cognitiva y de desarrollo de la lengua ha sido más frecuente por parte de lingüistas que de psicólogos o psicolingüistas.

Sin duda, ha de atribuirse un sustrato innato a la realidad constatable de que la especie humana está capacitada para delinear cauces de percepción singulares que le permiten modos de conocimiento y conceptualización del mundo. Pero esa predeterminación debe remitirse a mecanismos biológicos muy esenciales en los que bien pudieran descansar los procedimientos funcionales de percepción (auditiva, visual, táctil) básicos ${ }^{2}$. A partir de ahí, el predominio corresponde a la variación y a la diversidad, sobre todo en la esfera del desarrollo de la habilidad lingüística. Pero también en la maduración cognitiva hay particularidades, hay casos y casos.

El principio racional y lógico de progreso en la cognición secuencializa primero la conceptualización de objetos y después la de acciones y cualidades. Hay sin embargo estudios descriptivos y experimentos controlados sobre el desarrollo en entornos idiomáticos del japonés y del coreano que evidencian una alteración en ese orden. Primero parecen conceptualizarse las acciones y después los objetos. El peso del contexto idiomático o del habla dirigida se hace patente en la ordenación de etapas de maduración cognitiva. Estudios sobre la ubicación del yo y la discriminación cognitiva de personas en casos de hijos únicos, de gemelos o de niños que son segundos, terceros o cuartos en sus respectivos contextos familiares demuestran la incidencia del entorno en la conceptualización. Algo similar a lo que ocurre en los experimentos sobre conceptualización espacial o temporal con niños mayas, ingleses, daneses y japoneses, que subrayan las diferencias de identificación mostrativa según las distinciones idiomáticas en cada caso (cfr. C. Sinha et alii (1999), P. Brown (2001), L. De León (2001), y S. Levinson (2001)).

Los mecanismos de percepción, aun siendo comunes, están condicionados en sus resultados inmediatos por factores de entorno (físico, social, idiomático, familiar) que son los que canalizan las conceptualizaciones primeras ${ }^{3}$. Ciertamente todos los humanos estamos

2 Los órganos de percepción innatos en su desarrollo molecular y celular dan soporte a las percepciones primarias que corresponden a la especie humana. Pero son los entornos particulares los que las convierten en interpretaciones conceptuales de qué se ve, qué se oye, qué se toca, qué se huele, qué se saborea (cfr. Elman et. alii, 1999: 23).

3 Con palabras de M. Bowerman y S. Levinson (2001: 9),

Children growing up in different cultures experience worlds that differ noy only in language, but in just about every facet of physical, social, and emotional experience. 
igualmente capacitados para percibir el mundo, pero las conceptualizaciones que sobre la realidad se operan, no son coincidentes. $Y$ esto es lo interesante cuando se aborda el proceso de desarrollo cognitivo. Y se vuelve crucial cuando se trata de la adquisición de la lengua. Si es indiscutible que todos los niños siguen un proceso y que todos hablan, lo que resulta curioso y pide pormenor es cómo lo consiguen de manera variada y desigual.

La diferenciación entre los dos desarrollos -cognitivo y lingüístico y la asunción de que resulta necesario considerarlos en su autonomía ha hecho posible la detección de interesantes relaciones entre estadios y aspectos de cognición y de habilidad lingüística ${ }^{4}$. Pero además ha servido para que comenzara a verse en su pertinencia la dimensión de la comprensión en la dinámica de la adquisición de la lengua.

Un grueso de las investigaciones han concebido la adquisición de la lengua ciñéndola exclusivamente a la habilidad expresiva, en consonancia con el principio aludido de que hablar una lengua es manejar su gramática. Pero la faceta de la comprensión es esencial por varios motivos y en distintos frentes. En primer lugar, porque el niño comprende antes de que se exprese, hasta tal punto que comprender es requisito necesario en la activación de la expresión. En segundo lugar, porque la comprensión asegura o recompone la conceptualización del mundo al ser elementos léxicos paulatinamente fijados los que refieren los objetos. En tercer lugar, comprender significa, sobre todo, ser receptor cabal de mensajes, con todas las ventajas para la activación de las reglas pragmáticas de la lengua. Los estadios de progreso en la comprensión son piedras de toque esenciales para valorar el desarrollo y asentamiento del caudal léxico y para sopesar aspectos pragmáticos como la modalidad, los valores sociales ligados a las palabras, o la destreza discursiva y narrativa del niño. Y, naturalmente, sobre la comprensión se asientan buena parte de los experimentos diseñados para investigar el desarrollo cognitivo ${ }^{5}$.

En la misma medida en que se han de introducir, diferenciándolas, las dos vertientes de la habilidad -comprensión y expresión-, en el estudio de la adquisición se ha de borrar, asimismo, la identificación del proceso de desarrollo lingüístico con, exclusivamente, la emergencia de la gramática ${ }^{6}$. El progreso en la estructuración y riqueza de construcciones lingüísticas está sujeto a un acervo léxico suficiente que las haga posible, y, a su vez, la desenvoltura léxica hunde sus raíces en un progreso fónico adecuado para expresarse. Los componentes fónico y léxico han de contemplarse en sus fases y peculiaridades como inte-

4 El volumen editado por M. Bowerman y S. Levinson (2001) contiene interesantes contribuciones que ponen el acento en la relación entre desarrollo cognitivo y desarrollo lingüístico. La perspectiva interlingüística adoptada permite valorar relevancias conceptuales y secuenciaciones adquisitivas distintas según el tipo de lengua. El capítulo aportado por D. Slobin sobre las categorías morfológicas o los compuestos por S. Levinson, P. Brown y L. de León sobre la conceptualización espacial entre niños mayas subrayan la incidencia del entorno idiomático en la organización conceptual, y sus implicaciones en el proceso de adquisición de las lenguas. Hasta el extremo de que Slobin admite abiertamente que cada tipo de lengua tiene su modo propio de pensar para hablar (thinking for speaking, cfr. Slobin, 1996). A este respecto, Bowerman y Levinson (2001: 10) señalan que

The recent revolution in our understanding of infant cognition has yet to have its full impact on the study of language adquisition. Similarly, the great increase in our understanding of how children learn languages that differ radically in structure and underlying categorization has yet to be fully exploited as a source of information about he processes of cognitive development.

5 Tanto es así que muchos autores no sabrían discernir si se trata de comprensión de palabras o de conceptualización del mundo (cfr. Bowerman y Levinson, 2001: 3 ss.).

6 Para una argumentación más pormenorizada sobre las consecuencias de esa visión limitada de la adquisición, cfr. Fernández Pérez (en prensa 1). 
grantes capitales del complejo proceso de adquisición de la lengua, y como estadios previos inexcusables para el conveniente desarrollo de la gramática.

Por último, es prioritario e imprescindible desterrar el código adulto conformado como referencia de estudio o de valoración del lenguaje infantil. Quizás no sea un tópico, pero sí que es modo de proceder muy arraigado aproximarse a la investigación del desarrollo de la habilidad partiendo de las unidades o de las estructuras reconocidas en la lengua adulta. Las singularidades que definen los códigos que se van perfilando en las distintas etapas quedan así desfiguradas o malinterpretadas si los procedimientos de análisis no se ajustan o si el enfoque valorativo no se corresponde con el nivel simbólico y de codificación. El respeto por los datos, su descripción detenida y pormenorizada, y la convicción de que en el lenguaje infantil hay COMUNICACIÓN EFECTIVA en un dinámica que combina elementos propios, conducirán a presupuestos metodológicos razonables que dosifiquen la teoria en razón de su adecuación observacional o, como mucho, descriptiva. Lo importante es descubrir cómo se van delineando simbologías y códigos y cómo se les atribuye potencial comunicativo.

\section{Lenguaje infantil y teoría lingüística}

Pueden reducirse a dos los estilos de aproximación de las teorías lingüísticas a los materiales del habla infantil. Hay, de una parte, planteamientos apriorísticos que tienen el objetivo de confirmar su validez o su potencial por su capacidad predictiva en avanzar características o rasgos que, posteriormente, se detectan en los datos estudiados. Y se dan, de otra parte, actitudes y modos de abordar el habla infantil contemplando los usos lingüísticos en su realidad. El ropaje teórico es sólo el básico requerido para, sobre la observación y el análisis de suficientes datos, perfilar patrones simbólicos con entidad y eficacia comunicativa. No es tan importante comprobar la adecuación de la teoría como reconocer el progreso de la habilidad a través de códigos y perfiles lingüísticos y cognitivos en distintos estadios. El principio rector es el hallazgo de peculiaridades y constantes definitorias de los patrones evolutivos, sin forzar los datos ni al código conformado del adulto -y habitualmente basado en la norma escrita-, ni a lo que son esquemas o rasgos teóricos excesivamente generales y, por tanto, alejados de las singularidades genuinas del habla infantil. Las teorías pueden confirmarse y, sin embargo, los datos en su nivel descriptivo teniendo en cuenta el sistema dinámico del niño no están ni analizados ni rentabilizados. El carácter emergente de la lengua comporta que su construcción sea paulatina y epifenoménica, con estadios y características no identificables con la lengua adulta ${ }^{7}$. M. Tomasello (2003a), en una de sus contribuciones clave para abordar el desarrollo de la lengua, considera que las unidades y las construcciones manejadas por los niños no se acomodan a hormas alejadas del uso como son las estructuras formales de la lengua adulta ${ }^{8}$, ya que, en su opinión,

P. Hopper en un sugestivo trabajo sobre la noción de gramática emergente señala que

Grammar, in this view, is not the source of understanding and communication but a by-product of it. Grammar is, in other words, epiphenomenal [...] its structure is always deferred, always in a process but never arriving, and therefore emergent (Hopper, 1998: 156).

8 Es lo que R. Langacker (1987) llama la falacia de la lista de reglas. Derivada de equiparar los métodos formales de descripción con las características que han de mostrar los hechos comunicativos reales. En lugar de este enfoque concentrado en reglas establecidas, se propone una aproximación tildada como bottom up, que concede relevancia especial a los elementos singulares en el proceso de desarrollo de la lengua (cfr. Langacker, 1999). Con palabras de S. Kemmer y M. Barlow (1999: ix-x) 
Children must still learn the individual linguistic items and constructions of the language into which they are born, an this requires them to master many and various concrete pieces of language and to make straightforward abstractions across them. To repeat: this must happen regardless of one's theory (Tomasello, 2003a: 195).

Los elementos del habla infantil constituyen unidades que no se corresponden con la gramática en sentido estricto. De ahí que Tomasello (2003b: 8) afirme que "to maintain the myth of "The Grammar" of a language as a coherent entity many interesting structures must simply be ignored". La investigación de cómo los niños adquieren la lengua tendrá garantías de fiabilidad si se estudian los datos reales y genuinos de desarrollo. El tema es factual y empírico antes que racional (cfr. Tomasello, 2003: 328).

\subsection{Propósito comunicativo y "construcciones" en el habla infantil}

Si bien el móvil de interacción social en el desarrollo lingüístico está fuera de duda, no obstante hay tratamientos del habla infantil que lo obvian -quizás por no atribuirle pertinencia-, y abordan la descripción de características "gramaticales" desde el prisma de un modelo teórico que simula la capacidad potencial. Lo importante es comprobar que el marco y los principios que se han modelado desde la teoría se ajustan a ciertas características de algunos datos. Pero no se contempla ni la sistematicidad de las propiedades ni su valor y sus repercusiones en el conjunto. Que en el lenguaje infantil se vayan perfilando códigos propios en las diferentes etapas, y en consonancia con factores cognitivos y de interacción social, no parece estar en el punto de mira de aproximaciones ligadas a presupuestos generativistas.

En trabajos como los de M. Llinàs i Grau (2002) y A. Ojea (1997) y (2002), entre otros, se constata el empeño en otorgar validez y rendimiento a la versión minimista del generativismo, lo que es prioritario a cualquier otro objetivo de ponderación conjunta o incidental de las propiedades destacadas. Dice Llinàs i Grau (2002: 20-21) a este respecto que las propuestas generativistas

dan cuenta de miles de construcciones infantiles [...] en la actualidad el estudio de la adquisición es el laboratorio natural para la corroboración o invalidación de propuestas teóricas - la adquisición del lenguaje cobra hoy en día una relevancia impensada en otros tiempos (el subrayado es mío)

Claro que el modo de dar cabida a estructuras del habla infantil como Pas casser en niños franceses, y Fix mommy shoe en niños ingleses, obliga a recurrir a la teoría de la proyección que parece ser no determina en las primeras etapas la categoría Sconc. Según Ojea (1997: 439),

La existencia de sujetos nulos en las primeras etapas de lenguas no pro-drop sería debida, por tanto, a que el niño no proyecta la categoría Sconc en esos casos; si no hay Conc tampoco será obligatoria la expansión léxica de su especificador, de ahí que las oraciones de infinitivo matriz puedan tener un sujeto nulo.

The bottom up property adds that the specific and idiosyncratic elements of the system are privileged over the general in the acquisition and operation of the system: the general arises out of the specific, and the specific is what is most directly taken from experience. 
Con una gramática fuente como la que prima en la visión generativista de la adquisición de la lengua ${ }^{9}$, es inevitable la servidumbre del código adulto. No sólo por los procedimientos analíticos manejados sino también por las apreciaciones respecto de las fases en que se halla el lenguaje infantil. Llinàs i Grau (2002: 10) habla de una "morfología verbal defectuosa". Ojea (1997: 442) se refiere a problemas de procesamiento del niño para acceder a la subordinación. Sin duda, la concepción de la gramática, no como previa en origen sino como producto consecuente, en el sentido de gramática epifenoménica y emergente de P. Hopper no daría lugar a tales prejuicios. Con palabras de Hopper (1998: 155):

Emergent Grammar is a conception of linguistic structure that proposes to bypass the problem of a fixed, prediscourse adult grammar, with its attendant problems of necessarily "degenerate" input for both child acquisition and adult maintenance of language, by recolocating structure, that is, "grammar", from the center to the periphery of linguistic communication. Grammar, in this view, is not the source of understanding and communication but a by-product of it. Grammar is, in other words, epiphenomenal.

Frente a este modo de abordar el progreso desde la descripción puntual de peculiaridades en el marco de la teoría del producto, hay otros enfoques comunicativos que conceden pertinencia y entidad al proceso en sus etapas, destacando construcciones genuinas y características propias en cada nive ${ }^{10}$. Sistematicidad y valor comunicativo son parámetros para reconocer estadios de avance y poder así calibrar niveles de desarrollo gramatical. Lo ciertamente relevante es el proceso en sus fases, en él descansa el conocimiento del lenguaje. El tipo de unidades y construcciones que intervienen en cada estadio y su frecuencia de aparición son indicadores de su sedimento. Ya que en definitiva, tal y como afirma $\mathrm{M}$. Tomasello (2003: 318), "linguistic knowledge and linguistic processing are really just different aspects of the same thing".

La gramática semántica inicial, ya diseñada por R. Brown (1973) a base de relaciones conceptuales básicas ${ }^{11}$, ha sido la referencia clave para que en investigaciones recientes se haya puesto de manifiesto la importancia del caudal léxico, pero sobre todo en lo que concierne a verbos, en el progreso construccional del niño ${ }^{12}$. Las predicaciones, desde sus versiones más elementales hasta las construcciones adultas, ofrecen en el lenguaje infantil el escaparate idóneo para aproximarse a la emergencia de la gramática con cierto rigor empírico. Las investigaciones descriptivas en esta linea (cfr. el riquísimo volumen editado por Tomasello y Merriman (1995), sobre todo Golinkoff et alii (1995), Tomasello (1995) y

9 A. Ojea (2002: 419) afirma explícitamente que

los mecanismos cognitivos del niño y del adulto son los mismos. Esto implica que existe un conocimiento innato de las categorías sintácticas [...] las diferencias entre el lenguaje infantil en la etapa telegráfica y el lenguaje adulto son diferencias de rasgos gramaticales, no de gramática.

10 En consonancia con la convicción de que "How children become competent users of a natural language is not a logical problem but an empirical problem" (Tomasello, 2003: 328)

11 R. Brown (1973: 173) reconoce ocho relaciones semánticas básicas, a saber: (1) agente-acción (nena come), (2) acción-objeto (compra gusanitos), (3) agente-objeto (mamá zapato), (4) acción-locativo (sienta silla), (5) entidad-locativo (balón calle), (6) possedor-posesión (mio payaso), (7) entidad-atributo (caja roja), y (8) demostrativoentidad (este cuento).

12 Los estudios longitudinales de E. Bates y J. Goodman (1999) sobre población infantil típica y atípica (contempla casos de "síndrome específico de desarrollo del lenguaje", de "sindrome de Williams" y de "síndrome de Down") proporcionan resultados evolutivos en diferentes etapas que manifiestan la clara incidencia inicial del léxico en la emergencia de la gramática y la posterior interrelación de los dos componentes. 
Rispoli (1995)) han proporcionado evidencias sobre fases procesuales decisivas en el paso de la gramática semántica a la gramática formal.

Al margen de que el niño extienda en mayor o menor medida la etapa holofrástica, e independientemente de que su estilo cognitivo sea sintético o analítico, el desarrollo construccional vendrá marcado por el avance gradual de estructuras semánticas a estructuras formales. En cualquier caso, es la eficacia comunicativa la que orienta las etapas del progreso, y la que marca de manera reiterada las constantes procesuales de la dinámica de emergencia. Las primeras emisiones en la etapa holofrástica constituyen mensajes complejos siendo las palabras o los bloques de palabras verdaderos enunciados comunicativos. Son ilustrativos los casos siguientes ${ }^{13}$

Lucía: 2 años, 11 meses

*LUC: sí xxx rumpintaresa [*][?].

rumpintaresa $=$ rompe Teresa

*MAR: noo \# no ze rompe [*]!

${ }^{*}$ LUC: ++ la silla rumpintaresa $\left[{ }^{*}\right]$.

Xaquín: 2 años, 7 meses

Elia coge la carta de Cecilia

*XAQ: avereto [*].

avereto $=$ a ver esto

act: le quita la carta a Elia

Cuando en la etapa denominada telegráfica se dan las primeras combinatorias de unidades, el niño parte de esquemas comunicativos básicos que imita ${ }^{14}$. Los ejemplos que a continuación se enumeran hacen patente el mantenimiento de la rección habitual:

Borja: 2 años, 10 meses

*BOR: no sé cuánto más!

*PIL: $<$ y como $>[/ /]$ de qué color es bobi?

*BOR: $<$ de azul $>$ [*].

de azul = azul $\$ S Y N$;

*PIL: azul \# un perro azul!

Artai: 2 años, 7 meses

*MON: ese si que era un bicho muy grande.

*ART: mi $\left[{ }^{*}\right]<$ que culebra muy grande $>\left[{ }^{*}\right]$.

$\mathrm{mi}=$ mira $\$ \mathrm{PHO} ;$ que culebra muy grande $=$ que culebra más grande $\$ S Y \mathrm{~N}$

13 Los ejemplos están tomados del corpus de habla infantil (grupo koiné) sobre una muestra de 50 niños (31 niños y 19 niñas) de entre 22 meses y cuatro años. Las transcripciones se han operado según el sistema CHILDES de B. MacWhinney (cfr. MacWhinney (1991)).

14 Es lo que Tomasello (2003: 307) llama operaciones sintácticas basadas en el uso, porque since they explicitly indicate that the child does not put together each of her utterances from scratch, morpheme by morpheme, but rather, she puts together her utterances from a motley assortment of different kinds of preexisting psycholinguistics units. 
Como se puede comprobar en los ejemplos aducidos, paulatinamente, se van dando procesos de ensayo y de ampliación a partir de las hormas imitadas, sea insertando, sea añadiendo nuevas unidades, razón por la que se ha catalogado como etapa de corta y pega (filling in / adding on, vid. Tomasello, 2003: 307 ss.).

Más que adquirir palabras, morfemas, frases, o relaciones, los niños asientan dichas unidades en expresiones. La gramática que perfilan es, pues, semántica, esencialmente comunicativa, $y$, al tiempo, basada en construcciones que se imitan en su uso. La diversidad construccional facilitará el sedimento de estructuras y el progresivo control del aparato formal y combinatorio. Posibilitará, en definitiva, el paso gradual de lo ensayado y reiterado en usos por su efectividad comunicativa a los esquemas abstractos, que van tomando depósito una vez que el niño organiza y elabora activamente las construcciones que canalizan los mensajes.

\subsection{Procesos de desarrollo de la gramática en el período infantil}

El hallazgo de hitos o de mecanismos sistemáticamente reguladores del avance construccional constata la presencia de procesos genuinos en la adquisición de la gramática. En un sentido parejo a lo que sucede en el desarrollo fónico ${ }^{15}$, la dinámica de emergencia de reglas gramaticales viene también vehiculada a través de mecanismos singulares en procesos como los de predicación, combinatoria, y abstracción de esquemas formales, entre otros (cfr. Fernández Pérez (en prensa 2)).

Las predicaciones semánticas iniciales tienen su sentido comunicativo garantizado por ser repetición de lo escuchado.

Gara: 3 años, 3 meses / Gonzalo: 3 años, un mes

*GON:<un mono $>[<]$.

*PIL: y donde viven los monos?

*MAN: xxx.

${ }^{*}$ GON: <los árboles> [*].

los árboles = en los árboles $\$ S Y N$;

*GAR: <los árboles> [*].

los árboles = en los árboles \$SYN;

Ian: 2 años, 11 meses

*PIL: ese es pequeñito y este es la mamá \# si.

*IAN: <este e [*] mía [*]> [//] \# <este e mía mamá> [*]!

este e mía mamá $=$ esta mamá es mía

\%act: señala en la lámina

*PIL: este es de Ian y este es de Irene.

Al tiempo, el niño va asentando predicaciones básicas ligándolas a ciertas unidades, tal y como se ilustra en los siguientes casos:

15 Los procesos de adquisición fónica con reducción, asimilación, sustitución, auısencia o alteración de sonidos en los diferentes estadios reflejan el camino singular que los niños recorren en la sedimentación de las unidades fónicas (cfr. D. Ingram (1989)). 
Martín: 2 años, 11 meses

Borja: 2 años, 10 meses

*ANA:nadan \# quien sabe nadar?

*MAR: yo.

*BOR: yo.

*MAR: mira \# y yo poner [ $\left.{ }^{*}\right]$ las gafas y azi $\left[{ }^{*}\right]$.

poner = me pongo $\$ M O R ;$ azí = así $\$ \mathrm{PHO}$;

\%act: moviendo los pies como si estuviese nadando

*ANA: a ver \# pones las gafas $+/ /$.

*BOR: pones [*] las gafas y nadas.

$\%$ err: pones = te pones \$MOR;

\%act: Borja y Martín están haciendo que nadan

María: 3 años, un mes

*MON: lo pasáis mal conmigo?

*MAR: < yo> [/] yo <paso> [*] bien contigo.

paso = lo paso $\$ S Y N$;

Martín: 2 años, 9 meses

*PIL: quién vio alguna vez un caballo?

*MAR:<yo $>[>]$ !

*DAV: $<$ yo $>[<]$ !

*PIL: y dónde?

*MAR: <la caza $\left[{ }^{*}\right]$ la abu(ela)> $\left[{ }^{*}\right][/ /]$ yo vi a una mu@o <la [?] caza $\left[{ }^{*}\right]$ la abuela $>[*]$.

caza $=$ casa $\$ P H O$; la casa la abuela $=$ en la casa de la abuela $\$ S Y N$

Los mecanismos de sustitución o de inserción de nuevos segmentos constituyentes facilitan la ampliación construccional más allá de lo imitado. Si en una primera fase la habilidad conceptual-denominativa lleva a extender los usos de un ítem para distintas predicaciones relacionadas, en este estadio la soltura formal en el dominio de construcciones utilizadas en expresiones concretas provoca su ensayo en otros contextos. Los resultados pueden ser más o menos felices, como evidencian los ejemplos:

Xacobo: 3 años, 11 meses

*XAC: un día fue $\left[{ }^{*}\right]$ a una casa que no era mía \# a $<$ mirar en $>\left[{ }^{*} \mid\right.$ un caballo.

*CHE: si?

*XAC: a <mirar en> [*] un caballo que no era mío.

Martina: 3 años, 7 meses

*ANA: bueno \# pues vamos a la plaza \# y vamos a comprar un poco.

*MAR: sabías que $<$ mi mama $\left[{ }^{*}\right]>[/ /]$ mi papa $\left[{ }^{*}\right]$ tabaja $\left[{ }^{*}\right]<$ en las vacas $>[*]$.

en las vacas $=$ con las vacas $\$ S Y N$;

*ANA: trabaja con las vacas \# tu papá?

Adrián: 3 años, 11 meses

*DAV: sí \# no ves que tiene la cabeza y las alas.

*PIL: é un pescado \# chámase bacalao \#< a ver $>[>]$ ! 
*ADR: < claro < porque $>[/]<$ porque es como iguales $>[*]>[<]$.

porque es como iguales $=$ es igual al mío $\$ S Y N$

Hay que contar, además, con el incremento del caudal léxico y con la correspondiente repercusión en el aumento y la complejidad de las predicaciones. Las clases de verbos manejados y su aparición en construcciones con un número determinado de participantes serán piedras de toque esenciales en el análisis procesual de los procedimientos de rección. A continuación, se ofrecen construcciones en las que la complejidad de actantes provoca dudas e incluso ensayos en el orden de constituyentes:

Maria: 2 años, 9 meses

*MAR: $\quad<x x x$ no nos dejan $>[>]<$ los pañales no $>[/ /]<$ a los pañales los bebés> $\left[{ }^{*}\right]$ no nos dejan.

a los pañales los bebés $=$ los pañales de los bebés $\$ S Y N$;

*MAR:<los pañales los bebés $>\left[{ }^{*}\right]$ no nos dejan.

los pañales los bebés $=$ los pañales de los bebés \$SYN

Jorge: 3 años, 2 meses

*JOR: que <un dragón sale por la boca un fuego $>\left[{ }^{*}\right]$.

un dragón sale por la boca un fuego = al dragón le sale por la boca fuego $\$ S Y N$

Conforme se avanza en el uso de esas construcciones ligadas a expresiones imitadas, pero con inserciones, añadidos o sustituciones de constituyentes, el rápido incremento léxico promueve operaciones de combinatoria y asociación que exigen la emergencia de cierto control y planificación en las emisiones. De este modo se hace patente la importancia del léxico en el progreso de la gramática ${ }^{16}$. La ordenación de los constituyentes, el papel que se les asigne, o la determinación de los elementos, son indicios de emergencia formal de procesos de combinatoria. Como los que se observan en los ejemplos que siguen:

Iago: 2 años, 8 meses

*MON: y esto qué es?

*IAG: un [*] camixeta [*].

un = una $\$ M O R$; camixeta $=$ camiseta $\$ P H O$;

*MON: si.

Xacobo: 3 años

*XUL: las tengo rotas

$\%$ com: se refiere a las uñas

*XUL: las tengo rosas?

*TAT: pues las mías mira $\neq$ cochinas

*PIL: están cochinas?

\%act: riéndose

*PIL: y las de Xacobo están cochinas, están cochinas las de Xacobo?

*XAC. no las mías están pintadas <marronas> *marrón

*marronas $=$ de marrón.

16 En opinión de Tomasello (2003: 318) el léxico no sólo desempeña un papel importante para comprender la conceptualización lingǘstica del mundo en las primeras etapas, sino que es parte integral del desarrollo formal de la lengua en los distintos estadios. 
César: 3 años, 6 meses

*CES: y después condució $\left[{ }^{*}\right]$ y estaba <aquí en el co(che)> $[/ /]$ aquí en la moto.

condució = condujo \$MOR;

*CES: y después el coche condució $\left[{ }^{*}\right]$ y condució $\left[{ }^{*}\right]$ y no cayó.

condució = condujo \$MOR;

Víctor: 3 años, 3 meses

*MAR: <y le hacemos azí [*] con loz [*] huevoz [*] abilos $\left.{ }^{*}\right]>[<]$.

azí $=$ así $\$ \mathrm{PHO} ;$ loz $=\operatorname{los} \$ \mathrm{PHO} ;$ huevoz $=$ huevos $\$ \mathrm{PHO} ;$ abilos $=$

abrirlos \$PHO;

*VIC: y yo \# comi huevitos y me los tomí [*] con pan.

tomí = tomé \$MOR

Manuel: 3 años, 4 meses

*ANA: quién fue en caballo alguna vez?

*MAN: < yo> [/] yo fui <a un caballo> [*] muy pequeñito.

a un caballo $=$ en un caballo $\$ S Y N$

Si en las fases iniciales de la adquisición los niños manejan expresiones con valor comunicativo (y no palabras o categorías), progresivamente, en niveles posteriores de desarrollo, van delineando esquemas formales abstractos que le permiten planificar y controlar las emisiones. La selección activa de estas estructuras se hace patente en los mecanismos morfológicos y sintácticos ajustados, así como en la habilidad del niño para recomponer sobre la marcha la línea discursiva. Veamos algunos ejemplos:

Martín: 3 años

*MAR: pues yo me disfacé [*] \# y mamá tiene unas gafas y yo me pono [*] unas \# y las gafas tienen una nariz y esto chas@o y estaba de payaso.

disfacé = disfracé $\$ P H O$; pono = puse $\$ M O R$;

act $\%$ : cuando dice chas mueve la mano por encima de la cabeza señalando una goma o diadema imaginaria

Xulián: 3 años, un mes

*XUL: pues yo [?] era un tigre que había $<$ y le $>[/ /]<$ y le estaba $>[/ /]$ y le iba a clavar una lanza y se cayó tarzán y \#abía un agujero y se murió el tigre.

\%act: Xulián mueve la mano con fuerza para enfatizar

*ANA: ah se murió el tigre pero tarzán no.

*ANA: y tarzán salva a la chica la salva?

\%act: XUL dice que no con la cabeza

Víctor: 3 años, 2 meses

*MAR: <tiene> [//] y tiene un palo.

*ANI: un palo \# en la trompa \# claro.

*VIC: no \#<es> [/] es un tronco \# pero de un árbol \# que se rompió [*] \# que lo cortaron y $<$ ve $>[/ /]$ venió $\left[{ }^{*}\right]$ el elefante $<$ y lo $>[/]$ y lo

atrapalló@c.

rompió = rrompió $\$ \mathrm{PHO} ;$ venió $=$ vino $\$ \mathrm{MOR}$ 
La imagen plástica de elasticidad (bootstrapping) que en diferentes ocasiones se ha dibujado para reflejar la progresión de una gramática semántica a una gramática formal aparece coloreada por el papel que desempeña el léxico predicativo y por la incidencia de mecanismos formales que paulatinamente se van controlando. En opinión de M. Rispoli (1995), los niños no aprenden el significado de los verbos per se sino que van delineando los contenidos de las predicaciones -si son estados, actividades, procesos, causativas- y sobre esta base edifican representaciones primero semánticas y posteriormente sintácticas. Lo que quiere decir que se sirven de información pragmática y discursiva para identificar los distintos esquemas formales y para lograr diferenciar los posibles significados de una misma forma verbal.

\section{Recapitulación. ¿Qué es decisivo en el proceso de adquisición para que la dinámica del desarrollo tenga relevancia?}

La capacidad lingüística común a los humanos sólo se manifiesta si, progresivamente, la habilidad verbal va permitiendo al niño cubrir sus necesidades de interacción y comunicativas. Desde los primeros meses, los bebés manifiestan su tendencia a la comunicación, sea en sus protestas, sus miradas, o sus señalizaciones, sea en su atención a juegos, a llamadas y a conversaciones de "habla dirigida" de los adultos. El desinterés o la escasa concentración en los intercambios afectivo-comunicativos puede ser síntoma ya en esta primera etapa de disfunciones como el autismo. Del mismo modo que la ausencia de emisiones sonoras idiomáticas como continuación del balbuceo puede ser significativa y alertar de problemas auditivos.

El desarrollo de la habilidad verbal, paulatino y evolutivo (y no instantáneo y fijo) se conduce en componentes y etapas con características peculiares sistemáticas. La presencia de rasgos propios en cada nivel define los códigos comunicativos configurados según la etapa. El avance, estancamiento, o retraso en el proceso de adquisición de la lengua depende de si se han superado o no dichas fases. No parece suficiente afirmar sin más que el conocimiento del lenguaje es el mismo en todos los niños. Lo importante es comprobar que las habilidades comunicativas no coinciden y que, en muchas ocasiones, hay una distancia notable entre ellos. Detectar los desequilibrios a lo largo de la dinámica del desarrollo es garantía de poder remontar inconvenientes y dificultades en la adquisición del lenguaje. El proceso, aunque natural, está sujeto a condicionantes contextuales e individuales de muy distinto cariz. La pertinencia de estos factores es indudable para valorar y comprender los hitos de la trayectoria adquisitiva. Hitos que, en cualquier caso, muestran una enseña sobre todo comunicativa.

\section{Referencias bibliográficas}

Bates, Elisabeth y Goodman, Judith C. (1999): "On the Emergence of Grammar From the Lexicon", en B. MacWhinney (ed.), págs. 29-81.

Barlow, Michael y Kemmer, Suzanne (eds.) (1999): Usage based models of language, Stanford, Center for the Study of Language and Information.

Bowerman, Melissa y Levinson, Stephen C. (eds.) (2001): Language acquisition and conceptual development, Cambridge University Press. 
Bowerman, Melissa y Levinson, Stephen (2001): "Introduction", en M. Bowerman y S. Levinson (eds.) (2001), págs. 1-16.

Broeder, Peter y Murre, Jaap (eds.) (1999): Language and Thought in Development. Cross-Linguistic Studies, Tübingen, Gunter Narr.

Brooks, Patricia y Tomasello, Michael (1999): How young children constrain their argument structure constructions, Language, 75, págs. 720-738.

Brown, Robert W. (1973): A first language: the early stages, Cambridge, The Harvard University Press.

Brown, Penelope (2001): "Learning to talk about motion UP and DOWN in Tzeltal: is there a language-specific bias for verb learning?", en M. Bowerman y S. Levinson (eds.) (2001), págs. 512-543.

De León, Lourdes (2001): "Finding the richest path: language and cognition in the acquisition of verticality in Tzotzil (Mayan)", en M. Bowerman y S. Levinson (eds.) (2001), págs. 544-565.

Elman, Jeffrey L.; Bates, Elizabeth A.; Johnson, Mark H.; Karmiloff-Smith, Annette; Parisi, Domenico y Plunkett, Kim (1999): Rethinking Innateness. A Connectionist Perspective on Development, Cambridge, A Bradford Book, The MIT Press.

Fernández Pérez, Milagros (2003): "Pragmática y adquisición de la lengua", en Muñoz Núñez, M. et alii (eds.) (2003), págs. 1045-1056.

Fernández Pérez, Milagros (en prensa 1): "Adquisición del lenguaje y componentes de la lengua", en Actas del V Congreso de Lingüistica General (León 2002)

Fernández Pérez, Milagros (en prensa 2): "Algunos lugares comunes en el desarrollo de la Gramática", Revista Española de Lingüística.

Golinkoff, Roberta; Hirsh-Pasek, Kathy; Mervis, Carolyn B. y Frawley, William (1995): "Lexical Principles Can be Extended to the Acquistion of Verbs", en M. Tomasello y W. Merriman (eds.) (1995), págs. 185-221.

Gumperz, John y Levinson, Stephen (eds.) (1996): Rethinking linguistic relativity, Cambridge, Cambridge University Press.

Hopper, Paul J. (1998): "Emergent Grammar", en M. Tomasello (ed.) (1998), págs. 155-175.

Ingram, David (1989): First Language Acquisition. Method, Description, and Explanation, Cambridge University Press.

Kemmer, Suzanne y Barlow, Michael (1999): "Introduction: A Usage-Based Conception of Language", en M. Barlow y S. Kemmer (eds.) (1999), págs. vii-xxviii.

Langacker, Ronald (1987): Foundations of Cognitive Grammar, vol. 1, Stanford, Stanford University Press.

Langacker, Ronald (1999): "A Dynamic Usage-Based Model", en M. Barlow y S. Kemmer (eds.) (1999), págs. 1-63.

Levinson, Stephen C. (2001): "Covariation between spatial language and cognition, and its implications for language learning", en M. Bowerman y S. Levinson (eds.) (2001), págs. 566-588.

Llinàs i Grau, Mireia (2002): "Caminando hacia la gramática: una perspectiva teórica", Cognitiva. Revista de Psicología Experimental. Vol. Especial dedicado al Desarrollo del lenguaje.

MacWhinney, Brian (comp.) (1991): The CHILDES project: Tools for analyzing talk, New Jersey, Erlbaum.

MacWhinney, Brian (ed.) (1999): The Emergence of Language, N. Jersey / N. York, Lawrence Erlbaum.

Merriman, William E. y Tomasello, Michael (1995): "Introduction: Verbs Are Words Too", en M. Tomasello y W. Merriman (eds.) (1995), págs. 1-18.

Muñoz Núñez, M. ${ }^{a}$ Dolores; Piñero-Alcalá, Ana Isabel; Fernández Smith, Gérard y Benítez Soto, Victoria (eds.) (2003): Actas del IV Congreso de Lingüistica General, vol. III, Cádiz/Alcalá, Servicio de Publicaciones de la Universidad de Cádiz/Servicio de Publicaciones de la Universidad de Alcalá. 
Ojea, Ana I. (1997): "Categorías funcionales y adquisición de la primera lengua: un análisis contrastivo", Revista Española de Lingüistica, 27.2, págs. 425-446.

Ojea, Ana I. (2002): "El desarrollo sintáctico en la adquisición de la primera lengua: análisis de la etapa telegráfica de una sujeto monolingüe de español", Revista Española de Lingǘstica, 31.2, págs. 413-430.

Rispoli, Matthew (1995): "Missing Arguments and the Acquisition of Predicate Meanings", en M. Tomasello y W. Merriman (eds.) (1995), págs. 331-352.

Sinha, Chris; Thorseng, Lis; Hayashi, Mariko y Plunkett, Kim (1999): "Spatial language acquisition in Danish, English and Japanese", en P. Broeder y J. Murre (eds.), págs. 95-125.

Slobin, Dan (1996): "From thought and language to thinking for speaking", en J. Gumperz y S. Levinson (eds.) (1996), págs. 70-86.

Slobin, Dan (2001): "Form-function relations: how do children find out what they are?", en Bowerman, Melissa y Levinson, Stephen C. (eds.) (2001), págs. 406-449.

Tomasello, Michael (1995): "Pragmatic Contexts for Early Verb Learning", en M. Tomasello y W. Merriman (eds.) (1995), págs. 115-146.

Tomasello, Michael y Merriman, William E. (eds.) (1995): Beyond Names for the Things. Young Children's Acquisition of Verbs, New Jersey, Lawrence Erlbaum.

Tomasello, Michael (1998): "Introduction: A Cognitive-Functional Perspective on Language Structure", en M. Tomasello (ed.) (1998), págs. vii-xxiii.

Tomasello, Michael (ed.) (1998): The New Psychology of Language. Cognitive and Functional Approaches to Language Structure, N. Jersey, Lawrence Erlbaum.

Tomasello, Michael (2000): Do young children have adult syntactic competence?, Cognition, 74, págs. 209-253.

Tomasello, Michael (2003a): Constructing a Language. A Usage-Based Theory of Language Acquisition, Harvard University Press.

Tomaselio, Michael (2003b): "Introduction: Some Surprises for Psychologists", en M. Tomasello (ed.) (2003), págs. 1-14. 